$$
\begin{gathered}
\text { CONF-9605/73--1/ } \\
\text { ANL/ASD/CP }-89600
\end{gathered}
$$

\title{
The APS Machine Protection System (MPS) *
}

\author{
R. Fuja, B. Berg, N. Arnold, G. Decker, B. Dortwegt, M. FergusonRECEIVED \\ N. Friedman, J. Gagliano A. Lumpkin, G. Nawrocki, X. Wang \\ Argonne National Laboratory, 9700 South Cass Ave., Argonne, IL 60439 USA \\ AUG 121996 \\ ๑.S TI
}

\begin{abstract}
The machine protection system (MPS) that protects the APS storage ring vacuum chamber from $x$-ray beams, is active. There are over 650 sensors monitored and networked through the MPS system. About the same number of other process variables are monitored by the much slower EPICS control system, which also has an input to the rf abort chain. The MPS network is still growing with the beam position limits detection system coming on-line. The network configuration, along with a limited description of individual subsystems, is presented.
\end{abstract}

\section{INTRODUCTION}

An overall description of the Advanced Photon Source (APS) machine protection system (MPS) was given by A. Lumpkin at the 1995 PAC conference (1). Highlights from this paper are repeated here for completeness.

The APS storage ring has a circumference of 1104 meters and is divided into 40 sectors. Each sector has a bending magnet (BM) $\mathrm{x}$-ray source and there will be 35 insertion device (ID) sources. At $100 \mathrm{~mA}$ of $7-\mathrm{GeV}$ stored beam, the vacuum chambers are passively safe to bending magnet radiation under normal cooling water flow conditions. For closed gap insertion devices, $10 \mathrm{~kW}$ x-ray beams generated by a missteered beam pose an immediate danger to certain parts of the vacuum chamber, on the order of a few ms. The MPS uses many different sensors to monitor storage ring conditions and beam steering parameters (2). If an unsafe condition arises, a fast beam abort is generated by removing the if drive to the klystrons. The beam then coasts inward to the scrapers and is lost in about $300 \mathrm{~ms}$. All fault conditions are latched and, in an improved version of the logic, all faults are time stamped.

* Work supported by U. S. Department of Energy, Office of Basic Energy Sciences, under Contract, No. W-31-109-ENG-38. 


\section{DISCLAIMER}

This repor was prepared as an account of work sponsored by an agency of the United States Government. Neither the United States Government nor any agency thereof, nor any of their employees, makes any warranty, express or implied, or assumes any legal liability or responsibility for the accuracy, completeness, or usefulness of any information, apparatus, product, or process disclosed, or represents that its use would not infringe privately owned rights. Reference herein to any specific commercial product, process, or service by trade name, trademark, manufacturer, or otherwise does not necessarily constitute or imply its endorsement, recommendation, or favoring by the United States Government or any agency thereof. The views and opinions of authors expressed herein do not necessarily state or reflect those of the United States Government or any agency thereof. 


\section{DISCLAIMER}

Portions of this document may be illegible in electronic image products. Images are produced from the best available original document. 


\section{THE MPS SYSTEM}

The MPS system is divided into three functional categories:

1. Different fault condition sensors check everything from water and vacuum to beam missteering.

2. Local MPS cards collect signals from various fault sensors and report to a main MPS control card. There is one local MPS card for every two sectors.

3. A main MPS card with decision-making capabilities acts as the interface to the rf system. This network carries $1-\mathrm{MHz}$ heartbeat signals over fiber cables to a main machine protection system (MPS) logic circuit that controls rf switches, removing the drive to the klystrons. This logic circuit also receives input from beam current monitoring circuits, giving it decision-making capabilities.

The network comprises 20 cells, called local MPS cards, located in oddnumbered sectors around the storage ring. Two types of cells exist: the earlier version has inputs for 8 heartbeat signals, and an improved version has 12 inputs and provides a time stamp of requested aborts. Input to an unused cell is manually disabled. When the heartbeats to all active inputs are received, a cell generates an output heartbeat that goes to collection points located near the main MPS control logic card. Outputs from collection points activate the main logic card. Inputs to a local MPS cell come from individual sensors, such as beam position limits detector electronics, or from summation cards that receive information from many sensors, such as water flow, etc. Heartbeats from sensors monitoring each sector are fed into the inputs of local a MPS cell. There are 70 cell inputs reserved for experimental area beamline front ends; 35 for BM beamlines, and 35 for ID beamlines. The EPICS control system also has an input to the main heartbeat collection point. Activating a button on the control screen will dump the beam.

\section{THE MAIN MPS CARD}

The main MPS card acts as the interface to the low-level rf system and is triggered by the loss of a heartbeat signal, the removal of one of the beam current information signals, or the beam current exceeding a maximum preset threshold. If stored beam is above $0.5 \mathrm{~mA}$, loss of a heartbeat signal will remove the lowlevel $\mathrm{rf}$ signal to the klystron drivers for $100 \mathrm{~ms}$. If beam is not below $0.5 \mathrm{~mA}$, the klystron high voltage power supplies are tripped after $100 \mathrm{~ms}$ and, if beam is below $0.5 \mathrm{~mA}$ after $100 \mathrm{~ms}$, the low-level $\mathrm{rf}$ is reapplied. From the time an abort is requested until the time the beam decayed to $0.8 \mathrm{~mA}$ was measured at 700 microseconds. As long as the heartbeat signal remains missing, the main MPS 
card will remove the $\mathrm{rf}$ for $100 \mathrm{~ms}$ every time beam exceeds $0.5 \mathrm{~mA}$. The loss of one of the beam current signals will remove rf until the signal returns.

\section{THE SENSORS}

A list of the sensors in use around the ring appears below.

\begin{tabular}{lc}
\multicolumn{1}{c}{ Sensor } & Quantity \\
Absorber Water Flow & 320 \\
Vacuum Chamber Water Flow & 76 \\
Storage Ring Flags & 10 \\
Isolation Valves & 80 \\
Beam Position Limits Detector & 7 \\
Experiment Hall Front Ends & 14 BMs \\
Experiment Hall Front Ends & 9 IDs
\end{tabular}

A variety of sensors verify that machine systems are within their limits. The cooling water for the absorbers and the vacuum chamber as well as the vacuum gate valves are monitored through a series of VME-based latch cards. The status of storage ring flags and beamline exit port vacuum valves is input to the latch cards. Two latch cards are required to collect one sector's worth of signals; 80 latch cards cover the ring. When all inputs to the latch cards are present, a $1-\mathrm{MHz}$ heartbeat is sent to a local MPS cell card.

The threat of a missteered $\mathrm{ID}$ beam or a BM beam greater than $100 \mathrm{~mA}$ has resulted in a series of devices which protect the vacuum chamber. Beam position limits detector (BPLD) cards (3) are VME based and receive signals from the rf BPMs. These cards can average beam passes in the ring and have offset capabilities. The card functions are controlled from an EPICS page. If stored beam is within limits, a heartbeat signal is sent to a local MPS cell card. Each insertion device requires its own BPLD electronics, and wire monitors also check for beam missteering and blow up. There are eight beam position wire monitors installed and monitored by the EPICS control system but not connected to the abort system. A total of 320 vacuum chamber surface resistance temperature monitors (RTMs) are planned. Output data from all the RTM sensors in a sector will be combined via electronics to produce a single heartbeat signal per sector; the same will be true for the wire monitor sensors. Wire monitor and RTM sensors will have separate inputs to the MPS system.

Experiment hall front ends are stand-alone in nature and generate their own heartbeat signal when satisfied. Each beamline has its own protection system and an input to the MPS system. Figure 1 shows a block diagram of a typical local MPS cell and how it is connected to the main machine protection logic card. 


\section{Schematic of the Storage Ring Machine Protection System(MPS)}

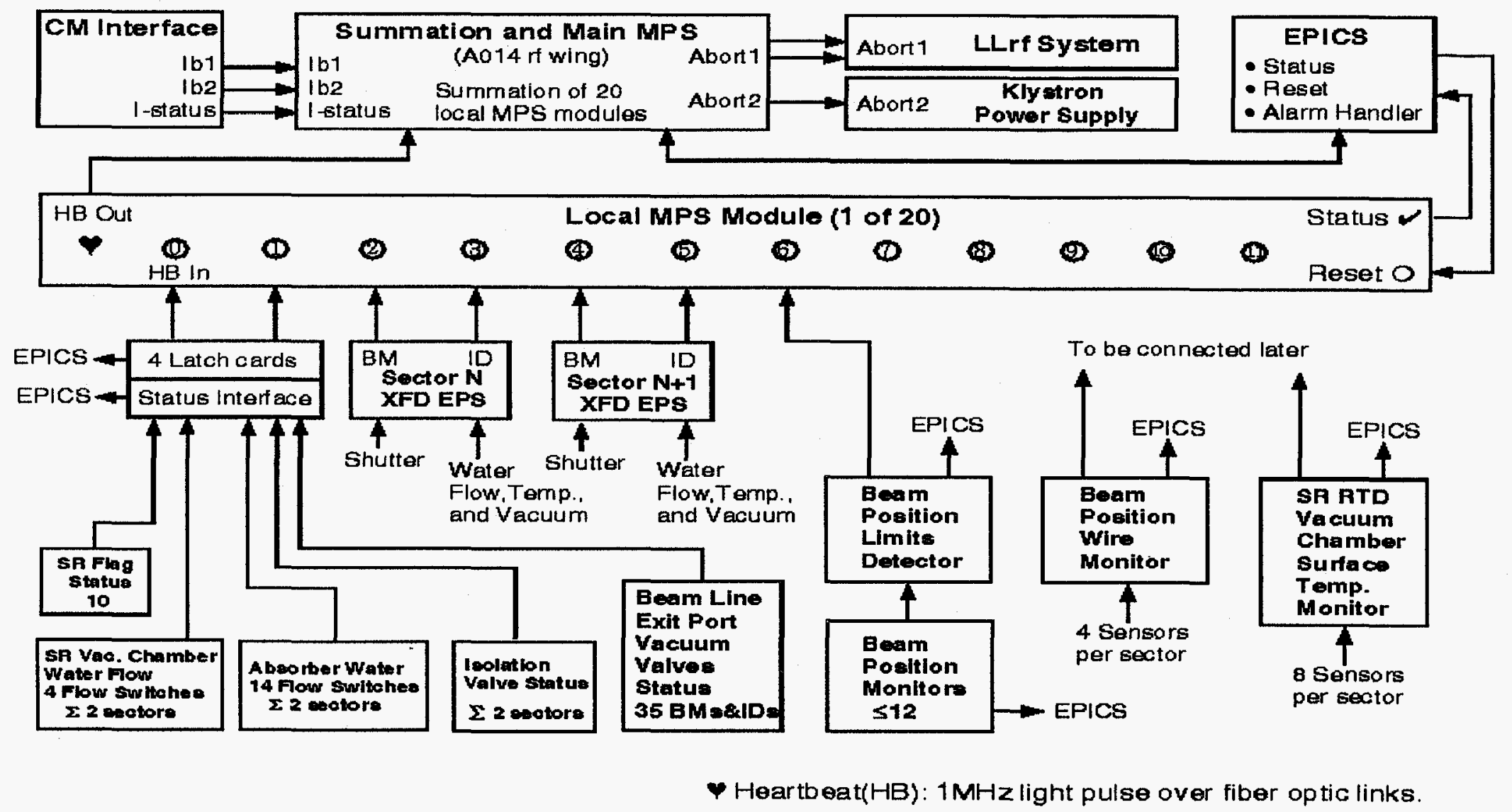

Figure 1 
A machine protection system cell for two sectors is shown in Figure 1, and 80 cells cover the storage ring. Heartbeat signals are carried over fiber optic cables, from latch cards and stand alone chassis to local MPS cells. When all activated inputs on a local MPS cell are present, the cell generates and transmits its heartbeat over fiber cables to a collection cell located next to the main MPS card. The main MPS card has digital outputs to the low-level if system and fiber outputs to the klystron power supplies.

\section{THE MPS CONTROL SCREEN}

Figure 2 shows a layout of the MPS control screen (4). This screen depicts the network and its interconnections and the status of individual sensors. Software beam limit conditions are imposed here. When the beam current transformer indicates beam conditions outside these limits, the EPICS input to the main MPS card is activated and latched. A software alarm handler can monitor any parameter in the control system and can activate the EPICS output to the main MPS card.

The 20 local MPS cells and the status of their inputs are displayed. As new systems come on-line, inputs to local cells are hardwired active. From this screen a postmortem of aborts is performed.

Verification of the entire MPS system is performed after a major shutdown, while verification of sector systems can be performed once sector work is complete. Every system connected to the MPS system must verify its performance to the next higher-level system. The beam position limits system must verify that the loss of its heartbeat is detected by its local MPS cell. The water and vacuum systems verify that they trip the latch cards they drive, and the latch cards must verify that they trip local MPS inputs. Each local MPS card input is cycled to verify that the main MPS card has been cycled to verify that the low-level rf goes off and the klystron high voltage power supplies trip on command.

\section{SUMMARY}

The MPS is active when beam is stored. The hardwired beam current limit can be set to a maximum of $300 \mathrm{~mA}$. Validation of beam position limits cards occurs before each run with beam current under $0.5 \mathrm{~mA}$. The wire monitor system and the RTM system are planned for future installation. The machine protection system as configured works well; there are very few nuisance aborts and they are being corrected. An upgrade to the main MPS card, now in progress, will allow for automatic testing and troubleshooting of this card. 


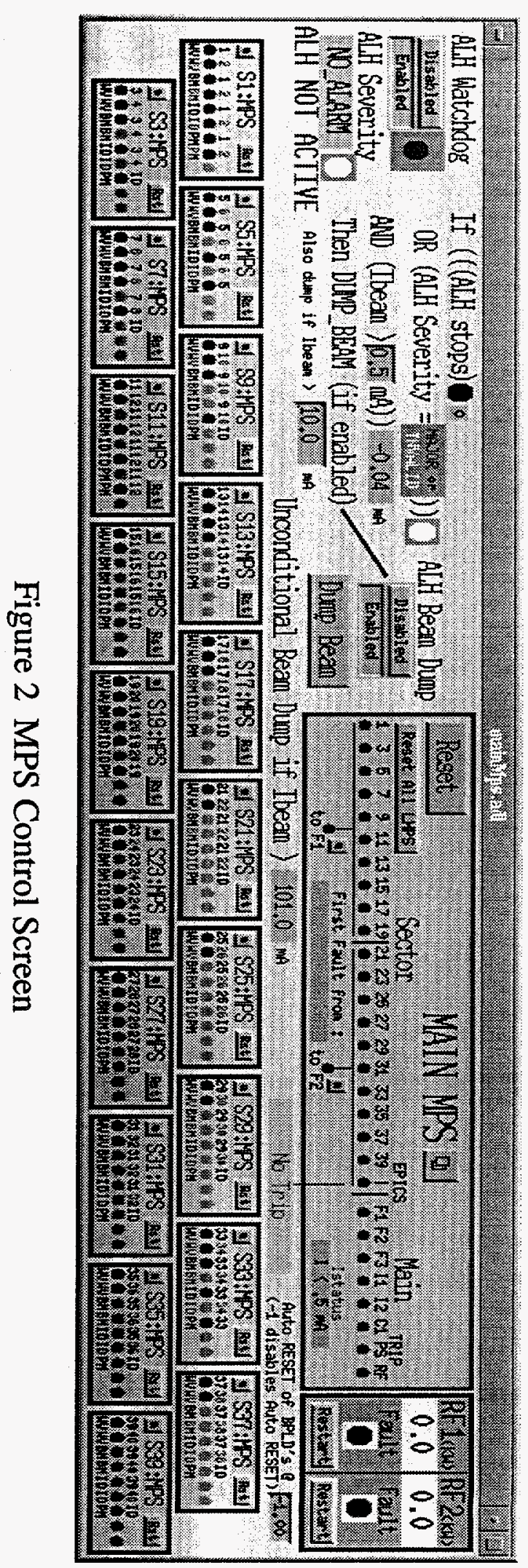




\section{REFERENCES}

1. A. Lumpkin et al., "Overall Design Concepts for the APS Storage Ring Machine Protection System," Proceedings of the 1995 IEEE PAC, 2467-2469 (1995).

2. G. Decker, "Abort Interlock Diagnostic for Protection of APS Vacuum Chamber," Proceedings of the 1993 IEEE PAC, Vol. 3, 2196 (1993).

3. X. Wang, private communication.

4. N. Arnold, private communication. 